\title{
The variable codons of H5N1 avian influenza A virus haemagglutinin genes
}

\author{
SHI WeiFeng ${ }^{1 \dagger}$, Mark J. GIBBS ${ }^{2}$, ZHANG YanZhou $^{3}$, ZHUANG DongMing ${ }^{4}$, DUN AiShe ${ }^{4}$, YU GuangFu ${ }^{4}$, \\ YANG NaNa ${ }^{1}$, Robert W. MURPHY ${ }^{5} \&$ ZHU ChaoDong ${ }^{3 \dagger}$ \\ ${ }^{1}$ Institute of Life Sciences, Taishan Medical College, Taian 271000, China; \\ ${ }^{2}$ School of Botany and Zoology, Faculty of Science, Australian National University, ACT 0200, Australia; \\ ${ }^{3}$ Institute of Zoology, Chinese Academy of Sciences, Beijing 100101, China; \\ ${ }^{4}$ Department of Basic Medicine, Taishan Medical College, Taian 271000, China; \\ ${ }^{5}$ Department of Natural History, Royal Ontario Museum, Toronto, ON M5S 2C6, Canada
}

We investigated the selection pressures on the haemagglutinin genes of H5N1 avian influenza viruses using fixed effects likelihood models. We found evidence of positive selection in the sequences from isolates from 1997 to 2007, except viruses from 2000. The haemagglutinin sequences of viruses from southeast Asia, Hong Kong and mainland China were the most polymorphic and had similar nonsynonymous profiles. Some sites were positively selected in viruses from most regions and a few of these sites displayed different amino acid patterns. Selection appeared to produce different outcomes in viruses from Europe, Africa and Russia and from different host types. One position was found to be positively selected for human isolates only. Although the functions of some positively selected positions are unknown, our analysis provided evidence of different temporal, spatial and host adaptations for H5N1 avian influenza viruses.

positive selection pressure, H5N1, avian influenza virus, HA, codon

Positive selection is defined as a significant excess of nonsynonymous $(\mathrm{dN})$ over synonymous $(\mathrm{dS})$ nucleotide substitutions ${ }^{[1]}$. In comparison, when negative selection occurred, selection acting on a pool of mutants might remove the mutations resulting in amino acids changes. In contrast, under positive selection, variants were preferred to replace the original population. In other words, positive selection pressure on them might favor the survival of new variants.

The haemagglutinin (HA) glycoproteins of influenza A viruses are partly exposed on virion surfaces and function to bind the virions to sialic acid-residues on host cell-surface receptors and then trigger membrane fusion permitting virus entry ${ }^{[2]}$, but they are also recognized by antibodies and they are the major surface antigen of the viruses. Mutations in H5N1 HA genes may favor the viruses' transmission from avian hosts to hu$\operatorname{mans}^{[3]}$.
Evidence of positive selection was first found in the HA genes, and in particular the codons encoding epitopes, of $\mathrm{H} 3$ and $\mathrm{H} 1$ subtype influenza A viruses infecting the human population ${ }^{[4,5]}$. Similarly, Campitelli et al. (2006) focused on the codons encoding one of the two HA cleavage products known as HA1, and found evidence of positive selection in the genes from isolates from 2001, 2003 and 2004, as well as evidence in the PB2 genes in 1997 and NS1 genes in $2004^{[6]}$. Smith et al. (2006) reported that only the M2 and PB1-F2 genes were positively selected in the H5N1 genotype $Z$ influenza viruses from southern China and Southeast Asia isolated from 2002 to $2005^{[7]}$. The number of positively selected sites in H5N1 from China and Southeast Asia decreased from 2004 to 2005, and no selection pressure

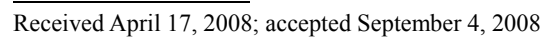
doi: 10.1007/s11427-008-0131-8

'Corresponding author (email: weifeng_cau@sina.com; zhucd@ioz.ac.cn)
} 
was found for $\mathrm{NS}^{[8]}$.

The methods that have been developed to detect evidence of selection pressure can be classified into three general classes: counting methods ${ }^{[9,10]}$, random effects modelling (REL) $)^{[11-13]}$ and fixed effects likelihood modelling (FEL) ${ }^{[14-16]}$. In FEL modeling, estimates of branch length and substitution rate models are fixed and the substitution rates for each site are fitted independently. In addition, a likelihood ratio test is done to test whether a site is under selection. One drawback of FEL modeling is that it is more computationally demanding and time-consuming than counting methods ${ }^{[14]}$. However, we selected FEL modeling for this study, because counting methods require a substantial number (e.g. $>50$ ) of sequences to gain power and REL is somewhat susceptible to Type 1 error for small datasets ${ }^{[17]}$.

Herein, we present a comprehensive study of selection pressure on H5N1 HA1 codons using the FEL method to evaluate the possible occurrence of adaptive changes that could favor interspecies virulence of avian influenza viruses. Our analysis provides evidence of time, region, and host-specific selection pressure acting on H5N1 HA1s.

\section{Materials and methods}

A dataset of HA sequences from $\mathrm{H} 5 \mathrm{~N} 1$ isolates from avian, mammalian hosts was complied from the GenBank database (http://www.ncbi.nlm.nih.gov/genomes/ FLU/FLU.html) directly. Copies of the dataset were sorted by isolation time, locality and host, respectively (Table 1).

Each subset was aligned using ClustalX $1.81^{[18]}$ with the default parameters. A Maximum Likelihood (ML) tree was built for each data subset using PhyML v2.4.4 for Windows ${ }^{[19]}$. Transition/transversion (Ts/tv) rate ratio and the proportions of invariable sites were estimated for the model. All other parameters were set as default.

Site-by-site positive selection analysis was performed for each data subset using the FEL method in the HyPhy software Package ${ }^{[20]}$. ML trees obtained in the previous step were used in the analysis. The HKY85 model was selected as the best model of sequence evolution for the data. This model allowed unequal base frequencies and different transition and transversion rates ${ }^{[21]}$. A global $\mathrm{dN} / \mathrm{dS}$ ratio was estimated using a codon model obtained by combining the MG94 and HKY85 models. The MG94 model assumed that nucleotide frequencies were shared by all three codon positions ${ }^{[22]}$. A two-rate FEL model was applied allowing $\mathrm{dN}$ and $\mathrm{dS}$ to be adjusted across sites. $P$ values $\leq 0.1$ were considered to be significant. All statistical analyses were completed using SPSS 11.5.0 for Windows and Pearson correlation coefficients were calculated based on the $\mathrm{dN}$ data of each dataset.

Table 1 Datasets included into analysis ${ }^{\text {a) }}$

\begin{tabular}{cccc}
\hline & \multicolumn{2}{c}{ Avian and human influenza viruses } & \multirow{2}{*}{ Host } \\
\cline { 2 - 3 } & Time & Region & \\
\cline { 1 - 2 } $1997(33 / 15)^{\text {b) }}$ & Africa (98/29) & Birds (173) \\
$2000(18)$ & China (361/7) & Chicken (361) \\
$2001(44)$ & Europe (61) & Duck (268) \\
& $2002(40)$ & Hong Kong & Goose (131) \\
& $2003(51 / 2)$ & Russia (30) & Human (159) \\
Dataset & $2004(179 / 30)$ & $\begin{array}{c}\text { Southeast Asia } \\
(353 / 95)\end{array}$ & Mammals (20) \\
& $2005(299 / 27)$ & West Asia & \\
& $2006(329 / 51)$ & & \\
& $2007(47 / 24)$ & & \\
\hline
\end{tabular}

a) Analysis was based on the nucleotide sequences of HA1 (966 bp) and all the GenBank accession numbers were listed in the supplementary file. b) The first numeral in the parentheses was the number of sequences in the dataset and the second was the number of sequences isolated from humans. Except the datasets in the last column, datasets with only one number in the parentheses were consisted of sequences without human source.

\section{Results}

\subsection{Positive selection analysis of the datasets sorted by time}

Evidence of positive selection was discovered from viruses isolated from all years, except for 2000 (Table 2). Viruses isolated from 2003 to 2005 provided the strongest evidence of positive selection. Sequences from 2004 appeared to have seven positively selected sites. Five sites were indicated to be under positive selection in 2003 and four in 2005. Codon positions 138, 140, 141, 155 and 156 were positively selected in most years (Table 2). Selection reduced amino acid polymorphism at position 138; only Lys and Gln were found in 2007 with Gln being found in nearly $90 \%$ of the sequences. By contrast, amino acid polymorphism increased at position 140 from 2003 to 2005. Amino acid polymorphism changed slightly at positions 141, 155 and 156 during this period of time (Table 2). Some positions were indicated to be under positive selection during a particular year only, such as positions 123 and 212 in 2004, position 120 in 2006 and positions 94 and 154 in 2007. 
Table 2 Positively selected sites and their amino acid polymorphism ${ }^{\text {a) }}$

\begin{tabular}{|c|c|}
\hline Item & Positively selected sites and their amino acid polymorphism \\
\hline \multicolumn{2}{|l|}{ Time data } \\
\hline 1997 & 156(A17/S6/T9/V1) \\
\hline 2001 & 156(A29/S3/T12) \\
\hline 2002 & 138(H1/L6/N13/Q20); 140(K34/R5/S1) \\
\hline 2003 & 138(H2/L6/N2/Q41); 140(K33/N2/R13/S2/T1); 141(P6/S45); 155(D2/G1/N6/S42); 156(A28/S4/T19) \\
\hline 2004 & $\begin{array}{l}\text { 123(P7/S172); 138(H2/L22/N6/Q149); 140(E2/K132/N3/R31/S9/T2); 141(F1/P26/S152); 155(D1/N15/S163); } \\
\text { 156(A47/I1/S3/T128); 212(E5/K65/R109) }\end{array}$ \\
\hline 2005 & 140(D1/E1/K108/M2/N13/Q3/R70/S59/T42); 141(P80/S219); 155(D9/N153/S137); 156(A108/S2/T188/?1) \\
\hline 2006 & $120(\mathrm{G} 1 / \mathrm{N} 4 / \mathrm{S} 324) ; 138(\mathrm{H} 3 / \mathrm{L} 69 / \mathrm{N} 3 / \mathrm{Q} 254)$ \\
\hline 2007 & 94(D8/N33/S5/T1); 154(D25/G1/N21) \\
\hline \multicolumn{2}{|l|}{ Geographical data } \\
\hline Africa & 154(D81/G1/N16); 252(N88/Y10) \\
\hline \multirow[t]{2}{*}{ China } & 45(D344/E/2/N15); 53(K13/R348); 120(N6/R355); 123(A4/F4/P23/S330); 126(D16/E342/G1/K2); \\
\hline & $\begin{array}{l}\text { 133(A13/S348); 138(H19/L48/N16/Q278); 140(A1/E12/K100/M6/N10/R49/S53/T130); 141(P184/S177); } \\
\text { 156(A146/I1/T198/S15/?1); 188(A1/I36/K3/T321); 207(L10/Q351) }\end{array}$ \\
\hline Europe & $86(\mathrm{~A} 52 / \mathrm{I} 4 / \mathrm{V} 5)$ \\
\hline Hong Kong SAR & $\begin{array}{l}\text { 86(A102/D2/S1/V4); 129(L16/S90/V3); 140(K71/R28/S3/T7); 141(P13/S96); 156(A71/K1/S12/T25); } \\
\text { 189(K86/M1/R22) }\end{array}$ \\
\hline Southeast Asia & $\begin{array}{l}\text { 94(D200/N76/S71/V6); 138(H3/L116/M1/N1/Q232); 140(D1/E2/K202/N8/Q3/R50/S70/T14/V3); } \\
\text { 151(I349/T2/V2); 155(D6/N22/R1/S324); 156(A35/I1/S1/T316); 200(I73/V280); 209(L337/M2/S13/V1); } \\
\text { 212(E5/K150/R198) }\end{array}$ \\
\hline \multicolumn{2}{|l|}{ Host data } \\
\hline Birds & 86(A120/I5/S3/T3/V42); 126(D8/E164/V1); 138(H1/L20/N11/Q141); 140(K58/N2/R96/S11/T6) \\
\hline Chicken & $\begin{array}{l}\text { 121(C1/D2/S354/Y4); 138(H8/I2/L108/M1/N8/Q234); 140(D1/E7/K154/M1/N5/R131/S27/T35); } \\
\text { 155(D14/N120/S227); 156(A148/I1/S16/T196); 171(D361) }\end{array}$ \\
\hline Duck & 138(H11/L24/N15/Q218); 156(A115/I1/S7/T145); 174(I82/V186); 212(E16/K205/R43/T4) \\
\hline Goose & $\begin{array}{l}\text { 138(H10/L14/M1/N2/Q104); 140(A1/E3/K40/M4/N1/R20/S13/T49); 156(A56/S3/T71/?1); } \\
\text { 309(D10/N113/S8) }\end{array}$ \\
\hline Human & 83(A114/I40/P2/T2/V1); 140(E2/K44/Q1/R61/S45/T6); 155(D3/N47/R1/S108); 156(A51/S5/T103) \\
\hline Mammals & 138(H1/L2/Q17); 140(K9/N1/R8/S2) \\
\hline
\end{tabular}

a) Sites are numbered according to amino acid positions starting from the methionine codon. The different amino acids present at each site under positive selection are given in parentheses. Following them is the number of sequences of each amino acid in the dataset respectively.

The $\mathrm{dN}$ values for the sequences from consecutive years were usually more similar than $\mathrm{dN}$ values from years far apart, as was shown by correlation coefficients (Table 3), and this was particular marked in the later years in the series. Correlation coefficients steadily became smaller as the separation of the datasets in time increased. Correlation coefficients computed between 2007 and any other years into analysis were the smallest, except in 1997 (Table 3).

\subsection{Positive selection analysis of the datasets sorted by regions}

Viruses from every region, except from Russia and West
Asia, gave evidence of positive selection. H5N1 viruses from southeast Asia and China yielded the strongest evidence with nine and 12 positively selected sites, respectively (Table 2). In these two geographic regions, the greatest degree of amino acid polymorphism occurred at position 140, with nine and eight different amino acid residues, respectively. Six positions were also probably under positive selection for viruses from Hong Kong, China.

Amino acid polymorphism varied geographically. Viruses from China commonly had Thr, Lys or Arg at position 140, whereas those from southeast Asia commonly had Lys, Ser or Arg, with few isolates processing 
Thr $\left(\chi^{2}=127.956, P=0.000\right)$. Similarly, about $90 \%$ of the Southeast Asia viruses possessed Thr at position 156, while only about half of the China isolates had Thr and $40 \%$ had Ala $\left(\chi^{2}=106.927, P=0.000\right.$; Table 1$)$. Some sites provided evidence of positive selection in certain areas but not others, such as site 252 in Africa, sites 45, 53 in China, site 189 in Hong Kong and site 94 in Southeast Asia (Table 2).

Table 3 Correlation coefficients between any two datasets from different years

\begin{tabular}{lllllllll}
\hline & 2000 & 2001 & 2002 & 2003 & 2004 & 2005 & 2006 & 2007 \\
\hline 1997 & $0.315^{\text {a) }}$ & 0.475 & 0.496 & 0.553 & 0.450 & 0.471 & 0.474 & 0.333 \\
2000 & & 0.469 & 0.420 & 0.482 & 0.505 & 0.445 & 0.406 & 0.328 \\
2001 & & & 0.683 & 0.704 & 0.649 & 0.574 & 0.521 & 0.356 \\
2002 & & & & 0.821 & 0.700 & 0.637 & 0.513 & 0.369 \\
2003 & & & & & 0.801 & 0.769 & 0.639 & 0.482 \\
2004 & & & & & & 0.787 & 0.682 & 0.498 \\
2005 & & & & & & & 0.831 & 0.574 \\
2006 & & & & & & & & 0.625 \\
\hline
\end{tabular}

a) The values were calculated based on $\mathrm{dN}$ data from positive selection analysis and were significant at the 0.01 level (2-tailed) if there was no special statement.

The $\mathrm{dN}$ values of the sequences from China, Hong Kong and southeast Asia correlated more strongly than those from any other two other regions (Table 4). Africa and Europe had higher correlation coefficients than Africa had with all other regions in the analysis. Europe had its highest correlation with China (Table 4). Correlation coefficients for Russia were highest with Europe. It should also be noted that, except for Russia, correlation coefficients from comparisons of the values with those from West Asia sequences were the lowest (Table 4).

Table 4 Correlation coefficients between any two datasets from different regions ${ }^{\text {a) }}$

\begin{tabular}{|c|c|c|c|c|c|c|}
\hline & China & Europe & $\begin{array}{l}\text { Hong } \\
\text { Kong }\end{array}$ & Russia & $\begin{array}{c}\text { Southeast } \\
\text { Asia }\end{array}$ & $\begin{array}{l}\text { West } \\
\text { Asia }\end{array}$ \\
\hline Africa & $0.332^{\text {a) }}$ & 0.423 & 0.326 & 0.363 & 0.314 & 0.311 \\
\hline China & & 0.539 & 0.750 & 0.241 & 0.787 & 0.208 \\
\hline Europe & & & 0.497 & 0.357 & 0.498 & 0.206 \\
\hline Hong Kong & & & & 0.215 & 0.718 & 0.193 \\
\hline Russia & & & & & 0.153 & 0.332 \\
\hline $\begin{array}{l}\text { Southeast } \\
\text { Asia }\end{array}$ & & & & & & $0.125^{\mathrm{b})}$ \\
\hline
\end{tabular}

a) The values were calculated based on $\mathrm{dN}$ data from positive selection analysis and were significant at the 0.01 level (2-tailed) if there was no special statement. b) This value was significant at the 0.05 level (2-tailed).

\subsection{Positive selection analysis of the datasets sorted by hosts}

HA1s from all types of hosts appeared to be positively selected (Table 2). Viruses from all birds gave evidence of four positively selected sites in the HA1s, while those from chickens, ducks and geese had 6, 4 and 6, respectively (Table 2). Position 138 appeared to be positively selected in all isolates except those from humans. Similarly, position 140 was positively selected for viruses of all host types except ducks. Some positions were positively selected only for viruses isolated from certain types of hosts, such as positions 86 and 126 for viruses from all birds, 121 and 171 for viruses from chickens, 309 for viruses from geese and 83 for viruses from humans (Table 2).

The pattern of $\mathrm{dN}$ values suggested that all viruses from avian sources were under similar positive selection (Table 5). Generally, correlation coefficients calculated from comparisons of $\mathrm{dN}$ values from human isolates and other host types were relatively small and they were less than those between mammals and other hosts. Correlation coefficients between non-primate mammals and human were smaller than those between non-primate mammals and viruses of avian sources (Table 5).

Table 5 Correlation coefficients between any two datasets from different hosts

\begin{tabular}{llllll}
\hline & Chicken & Duck & Goose & Human & Mammals \\
\hline Birds & $0.741^{\text {a) }}$ & 0.819 & 0.826 & 0.651 & 0.730 \\
Chicken & & 0.870 & 0.774 & 0.588 & 0.660 \\
Duck & & & 0.846 & 0.655 & 0.682 \\
Goose & & & & 0.665 & 0.702 \\
Human & & & & & 0.653 \\
\hline
\end{tabular}

a) The values were calculated based on $\mathrm{dN}$ data from positive selection analysis and were significant at the 0.01 level (2-tailed) if there was no special statement.

\section{Discussion}

Our results suggested that H5N1 from avian, human and mammalian sources have been under positive selection during most of the years considered, and especially since 2003. During this period of time, H5N1 influenza viruses continued to extend their geographical range from Hong Kong to Southeast Asia in 2004, to Middle East and some European countries in 2005 and to some African countries and Australia in 2006 (http://www.who.int/ csr/disease/avian_influenza/timeline_19_10_2007.pdf). 
Positive selection pressure acting on them might have promoted their geographical extension and frequent transmission to humans. The relatively low correlation coefficients calculated from comparisons of 2007 isolates with the other years suggested evolutionary pressure on HAs of the 2007 isolates might have changed.

Some sites positively selected in viruses from different regions displayed different amino acid patterns. For example, at position 140, near the antigenic site A, most Chinese isolates held $\mathrm{Thr}$ and Lys while those from Southeast Asia mainly maintained Lys. Likewise, the Ala156Thr mutation occurred in most of isolates from Southeast Asia, but only about half of the strains from China were found to have this mutation. Although these sites were positively selected in different regions, different amino acid patterns were produced to adapt to different circumstances.

Selection pressure on isolates from neighboring regions were often more similar than those from distant regions. This might be caused by comparable geographical pressures and migration routine. However, with respect to selection pressure, viruses from Africa, Europe and West Asia were more similar to those of Russia. Additionally, the H5N1 viruses isolated from these four regions appeared to be unusually stable for nearly a year ${ }^{[23]}$. Therefore, this similarity likely reflects migratory patterns of $\mathrm{H} 5 \mathrm{~N} 1$ viruses in wild birds from Russia to these regions. Remarkably, West Asia strains experienced different selection pressures compared to those of adjacent regions, such as China. These viruses have likely evolved to better adapt to local circumstances.

A zoonotic virus must overcome a series of obstacles in order to infect and successfully transmit from one person to another ${ }^{[24]}$. Mutations at some sites might facilitate human-to-human infection. However, human H5N1 infections were considered to be caused by avian

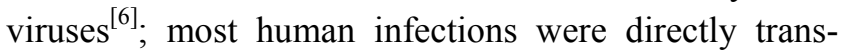
mitted from birds and few positions had a different amino acid pattern. We also discovered that humans and other mammalian $\mathrm{H} 5 \mathrm{~N} 1$ viruses experienced positive selection pressure similar to that of avian sources. Nonetheless, some sites were detected to be uniquely selected for different hosts. In particular, one site, position 83 , was found under positive selection and uniquely selected for human isolates. It was located near the position 84 , the antigenic site E. This site might be associated with an increased ability to neutralize human anti- bodies.

Positions 138, 140 and 141 had been identified either as targets of neutralizing antibodies, or as being involved in the carbohydrate recognition specificity of the HA towards the host-cell receptor ${ }^{[25]}$. Increased amino acid polymorphisms at position 140 from viruses from 2002 to 2005, China and Southeast Asia, as well as chickens and geese might suggest enhanced immunological pressure coming from extensive use of poultry vaccines.

Receptor specificity of influenza viruses, including H5, was not limited to the differential recognition of Siaa2,3-Gal and Sian2,6-Gal moieties, but appeared to be modulated more finely by certain residues in the vicinity of the receptor-binding sites ${ }^{[26,27]}$. Some sites verified in this study were located at or near the receptor-binding sites, as well as some more distant from them (Table 6). The positions that have not been reported before to be under positive selection are listed (Table 6). Although biological functions of some sites were still unknown, these sites might function as a whole to determine the ability of binding receptors from different hosts, pathogenicity and virulence of H5N1.

Table 6 Positively selected sites and their potential functional significance

\begin{tabular}{cl}
\hline Positively selected sites ${ }^{\text {a) }}$ & Potential functional significance \\
\hline 53 & Unknown \\
110 & Near antigenic site E \\
120 & Unknown \\
121 & Near antigenic site B \\
123 & Near antigenic site B \\
126 & Near antigenic site B \\
133 & Near antigenic site B \\
154 & Antigenic site A? Receptor binding? \\
171 & Glycosylation site \\
174 & Unknown \\
200 & Receptor binding? \\
207 & Unknown \\
209 & Near antigenic site D \\
252 & Near antigenic site D \\
309 & Unknown \\
\hline & Unknown \\
\hline & \\
\hline
\end{tabular}

a) Positions started from the mature $\mathrm{H} 5 \mathrm{HA}$ and only the sites unreported before were showed.

Mutations Asn182Lys and Gln192Arg were believed to convert independently the HAs of H5N1 viruses from 
recognizing avian receptors to human receptors ${ }^{[3]}$. In addition, the mutation to an Asn at position 223 slightly increased the SAa2,6Gal-binding capacity of the VN1194 HA $^{[3]}$. However, our results showed all the three sites were not under position selection. Only a few viruses isolated from humans after 2004 have one of the three mutations. Consequently, these mutations might not have resulted in advantages.

Amino acid sites have been used to trace the expansion of $\mathrm{H} 5 \mathrm{~N} 1$, including the three extensive migration events: Guangdong to Hong Kong SAR 1997, Guangdong to Thailand 2003 and Guangdong to Qinghai $2005^{[28]}$. We found that a few of these sites were under positive selection. This discovery might reveal the genetic factors that resulted in these thrusts in spatial expansion.

Numbers of positively selected sites found herein differed from those of other studies ${ }^{[6,8]}$. On one hand, this discrepancy might be caused by different methods used to infer positive selection. It should also be noted that we did not perform a model test procedure for the datasets but rather selected the default models in the HyPhy software package. However, if the applied nucleotide

1 Bush R M, Fitch W M, Bender C A, et al. Positive selection on the H3 hemagglutinin gene of human influenza virus A. Mol Biol Evol, 1999, 16: $1457-1465$

2 Webster R G, Bean W J, Gorman O T, et al. Evolution and ecology of influenza A viruses. Microbiol Rev, 1992, 56: 152-179

3 Yamada S, Suzuki Y, Suzuki T, et al. Haemagglutinin mutations responsible for the binding of $\mathrm{H} 5 \mathrm{~N} 1$ influenza A viruses to human-type receptors. Nature, 2006, 444: 378-382

4 Fitch W M, Leiter J M E, Li X, et al. Positive Darwinian evolution in human influenza A viruses. Proc Natl Acad Sci USA, 1991, 88: $4270-4274$

5 Ina Y, Gojobori T. Statistical analysis of nucleotide sequences of the hemagglutinin gene of human influenza A viruses. Proc Natl Acad Sci USA, 1994, 91: 8388-8392

6 Campitelli L, Ciccozzi M, Salemi M, et al. H5N1 influenza virus evolution: a comparison of different epidemics in birds and humans (1997-2004). J Gen Virol, 2006, 87: 955-960

7 Smith G J, Naipospos T S P, Nguyen T D. et al. Evolution and adaptation of H5N1 influenza virus in avian and human hosts in Indonesia and Vietnam. Virology, 2006, 350: 258-268

8 Ciccozzi M, Montieri S, Facchini M, et al. Evolutionary analysis of HA and NS1 genes of H5N1 influenza viruses in 2004-2005 epidemics. Avian Dis, 2007, 51(1 Suppl): 455-460

9 Nielsen R. Mapping mutations on phylogenies. Syst Biol, 2002, 51(5): model is not simple (e.g. F81) and allows ts/tv biases, then the model does not influence the estimates of sites under selection (Sergei L. Kosakovsky Pond, personal communication). On the other hand, our analysis included some small datasets and the increased numbers of available sequences might have led to greater statistical power, especially for small datasets including less than 50 sequences. After all, FEL tends to be sensitive to datasets of intermediate size ( $20-50$ sequences) and the results might become less repeatable.

We investigated the time-, region- and host-specificities of selection pressures acting on HA1s of H5N1 influenza viruses. Although the functions of some positions under positive selection remained unknown, our analysis provided evidence of different temporal, spatial and host adaptations for $\mathrm{H} 5 \mathrm{~N} 1$ avian influenza viruses. Monitoring the mutation events of $\mathrm{H} 5 \mathrm{~N} 1$ viruses of both avian and mammalian sources will be helpful discover sites involved in receptor recognition, pathogenicity and virulence.

The authors thank Dr Sergei L. Kosakovsky Pond in University of California, San Diego, for providing guidance on use of the HyPhy software package.

$729-739$

10 Suzuki Y, Gojobori T. A method for detecting positive selection at single amino acid sites. Mol Biol Evol, 1999, 16: 1315-1328

11 Huelsenbeck J P, Dyer K A. Bayesian estimation of positively selected sites. J Mol Evol, 2004, 58: 661-672

12 Nielsen R, Yang Z H. Likelihood models for detecting positively selected amino acid sites and applications to the HIV-1 envelope gene. Genetics, 1998, 148: 929-936

13 Yang Z H, Nielsen R, Goldman N, et al. Codon-substitution models for heterogeneous selection pressure at amino acid sites. Genetics, 2000, 155: $431-449$

14 Kosakovsky Pond S L, Frost S D W. Not so different after all: A comparison of methods for detecting amino acid sites under selection. Mol Biol and Evol, 2005, 22: 1208-1222

15 Suzuki Y. New methods for detecting positive selection at single amino acid sites. J Mol Evol, 2004, 59: 11-19

16 Yang Z, Swanson W. Codon-substitution models to detect adaptive evolution that account for heterogeneous selective pressures among site classes. Mol Biol Evol, 2002, 19(1): 49-57

17 Kosakovsky Pond S L, Frost S D W. Datamonkey: rapid detection of selective pressure on individual sites of codon alignments. Bioinformatics, 2005, 21(10): 2531-2533

18 Thompson J D, Gibson T J, Plewniak F, et al. The CLUSTAL_X windows interface: Flexible strategies for multiple sequence align- 
ment aided by quality analysis tools. Nucl Acids Res, 1997, 25: 4876-4882

19 Guindon S, Gascuel O. A simple, fast, and accurate algorithm to estimate large phylogenies by maximum likelihood. Syst Biol, 2003, 52: $696-704$

20 Kosakovsky Pond S L, Frost S D W, Muse S V. HyPhy: hypothesis testing using phylogenies. Bioinformatics, 2005, 21: 676-679

21 Hasegawa M, Kishino H, Yano T A. Dating of the human ape splitting by a molecular clock of mitochondrial-DNA. J Mol Evol, 1985, 22: $160-174$

22 Muse S V, Gaut B S. A likelihood approach for comparing synonymous and nonsynonymous nucleotide substitution rates, with application to the chloroplast genome. Mol Biol Evol, 1994, 11: 715-724

23 Normile D. Avian influenza: Evidence points to migratory birds in H5N1 spread. Science, 2006, 311: 1225

24 Webby R, Hoffmann E, Webster R. Molecular constrains to interspe- cies transmission of viral pathogens. Nature Med, 2006, 10 $\mathrm{S} 77-\mathrm{S} 81$

25 Kaverin N V, Rudneva I A, Ilyushina N A, et al. Structure of antigenic sites on the haemagglutinin molecule of $\mathrm{H} 5$ avian influenza virus and phenotypic variation of escape mutants. J Gen Virol, 2002, 83: $2497-2505$

26 Gambaryan A, Yamnikova S, Lvov D, et al. Receptor specificity of influenza viruses from birds and mammals: New data on involvement of the inner fragments of the carbohydrate chain. Virology, 2005, 334: $276-283$

27 Ilyushina N A, Rudneva I A, Gambaryan A S, et al. Receptor specificity of H5 influenza virus escapemutants. Virus Res, 2004, 100: $237-241$

28 Wallace R G, HoDac H, Lathrop R H, et al. A statistical phylogeography of influenza A H5N1. Proc Natl Acad Sci USA, 2007, 104: $4473-4478$ 